\title{
Extent of coronary atherosclerosis and arterial remodelling in women: the NHLBI-sponsored Women's Ischemia Syndrome Evaluation
}

\author{
Stephen J. Nicholls ${ }^{1}$, E. Murat Tuzcu ${ }^{2}$, Kathy Wolski ${ }^{3}$, B. Delia Johnson ${ }^{4}$, George Sopko ${ }^{5}$, Barry L. \\ Sharaf $^{6}$, Carl J. Pepine ${ }^{7}$, Steven E. Nissen ${ }^{3}$, C. Noel Bairey Merz ${ }^{8}$ \\ ${ }^{1}$ South Australian Health and Medical Research Institute (SAHMRI), Adelaide, South Australia, Australia; ${ }^{2}$ Cleveland Clinic Abu Dhabi, Abu Dhabi, \\ UAE; ${ }^{3}$ Cleveland Clinic, Cleveland, OH, USA; ${ }^{4}$ Graduate School of Public Health, University of Pittsburgh, Pittsburgh, PA, USA; ${ }^{5}$ National Heart, \\ Lung, and Blood Institute, Bethesda, MD, USA; ${ }^{6}$ Brown University, Providence, Rhode Island, USA; ${ }^{7}$ University of Florida, Gainesville, FL, USA; \\ ${ }^{8}$ Cedars-Sinai Heart Institute, Los Angeles, CA, USA \\ Contributions: (I) Conception and design: G Sopko, CJ Pepine, CN Bairey Merz; (II) Administrative support: CN Bairey Merz; (III) Provision of \\ study materials or patients: CJ Pepine, CN Bairey Merz; (IV) Collection and assembly of data: SJ Nicholls, EM Tuzcu, K Wlski, SE Nissen; (V) Data \\ analysis and interpretation: BD Johnson; (VI) Manuscript writing: All authors; (VII) Final approval of manuscript: All authors. \\ Correspondence to: C. Noel Bairey Merz, MD, FAHA, FACC. 127 S. San Vicente Blvd, Suite A3600, Los Angeles, CA 90048, USA. Email: merz@cshs.org.
}

Background: Information regarding the pathogenesis of ischemic heart disease (IHD) in women is limited. Sex-specific responses to atherosclerosis and coronary arterial remodelling in women versus men have been hypothesized, but limited study exists.

Methods: Case-matched study of 174 women with suspected ischemia referred for coronary angiography: 87 with non-obstructive coronary artery disease (CAD) (no luminal diameter stenosis $>20 \%$ in any coronary artery) and 87 age and ethnicity matched women with obstructive CAD. Groups were compared with regard to atheroma burden and coronary arterial remodelling assessed by coronary artery intravascular ultrasound (IVUS).

Results: IVUS revealed more extensive atheroma with obstructive CAD vs. those without obstructive CAD, with greater percent atheroma volume (PAV) $(36.1 \% \pm 9.8 \%$ vs. $25.4 \% \pm 9.1 \%, \mathrm{P}<0.0001)$, total atheroma volume (TAV) $\left(140.8 \pm 58.7\right.$ vs. $\left.98.8 \pm 46.9 \mathrm{~mm}^{3}, \mathrm{P}<0.0001\right)$ and percentage of images containing plaque $(70.0 \% \pm 30.5 \%$ vs. $35.7 \% \pm 32.6 \%, \mathrm{P}<0.0001)$. Adjusting for risk factors, PAV $(35 \% \pm 1 \%$ vs. $28 \% \pm 1 \%$, $\mathrm{P}=0.0008)$, TAV $\left(131 \pm 7\right.$ vs. $\left.115 \pm 7 \mathrm{~mm}^{3}, \mathrm{P}=0.110\right)$ and percentage of images containing plaque $(66 \% \pm 4 \%$ vs. $45 \% \pm 5 \%, \mathrm{P}=0.0008)$ remained greater with obstructive CAD. Obstructive CAD was associated with smaller lumen volumes $\left(251.9 \pm 92.8\right.$ vs. $\left.289.7 \pm 91.8 \mathrm{~mm}^{3}, \mathrm{P}=0.005\right)$, but surprisingly, the external elastic membrane (EEM) volume was very similar comparing the groups $\left(392.7 \pm 128.1\right.$ vs. $\left.388.6 \pm 113.7 \mathrm{~mm}^{3}, \mathrm{P}=0.910\right)$.

Conclusions: Our findings suggest that women referred to angiography for suspected ischemia, have differing patterns of coronary arterial response to injury with regard to accumulation of atherosclerosis and compensatory remodelling related to the presence and absence of obstructive CAD. Preservation and cultivation of compensatory arterial remodelling may be a novel CAD therapeutic target.

Keywords: Ischemic heart disease in women (IHD in women); coronary intravascular ultrasound (coronary IVUS); non-obstructive coronary artery disease (non-obstructive CAD); adverse coronary remodelling

Submitted Jan 05, 2018. Accepted for publication March 16, 2018.

doi: $10.21037 / \mathrm{cdt} .2018 .04 .03$

View this article at: http://dx.doi.org/10.21037/cdt.2018.04.03 


\section{Introduction}

Ischemic heart disease (IHD) is the leading cause of mortality in women at all ages in the Western world (1-3). When a diagnosis of IHD is established, women are less likely to be treated with established medical therapies and suffer greater adverse outcomes compared to men (4-8). Given an under-representation in clinical studies of IHD, little is known about the natural history and pathogenesis of atherosclerotic coronary disease in women (9).

A number of important distinctions between men and women have been noted. Women are more likely to present with atypical symptoms and have more functional disability from their symptoms (10). Rates of non-obstructive coronary artery disease (CAD) on angiography are higher in women with suspected ischemia $(10,11)$. In order to provide further insight into the factors that promote coronary heart disease (CHD) in women, the National Institutes of HealthNational Heart, Lung, and Blood Institute sponsored the Women's Ischemia Syndrome Evaluation (WISE) study (12). Investigating a cohort of women presenting for a clinically indicated coronary angiogram for suspected ischemia, a high rate of atherosclerosis, vascular dysfunction, ischemia and adverse events was observed in women without obstructive CAD $(10,13)$. This has led to the hypothesis that coronary vascular dysfunction in the setting of atherosclerosis with preserved epicardial coronary lumen dimensions, due to compensatory remodelling, may be a prevalent pattern of CAD in women with ischemia (13).

While sex-specific responses to arterial wall injury, with regard to atherosclerosis and epicardial arterial remodelling, have been hypothesized, little is known about the relationship between plaque accumulation and arterial remodelling in women. Interaction between atheroma burden and epicardial arterial remodelling is an important determinant of the degree of luminal obstruction for a given amount of plaque (14). The WISE intravascular ultrasound (IVUS) findings, although documenting a very high prevalence of atherosclerosis among a subgroup of symptomatic women without obstructive CAD by angiography (13), were limited by lack of a comparison with women having obstructive CAD.

Accordingly, to better understand the coronary remodelling process among women, this analysis was conducted using a case-control design. The objective was to characterize and compare coronary remodelling and extent of atherosclerosis and among women with and without obstructive CAD on angiography using IVUS, the most sensitive imaging modality for characterization of extent of atherosclerosis and patterns of arterial remodelling.

\section{Methods}

\section{Selection of subjects}

The WISE study (NCT00832702) enrolled women with chest pain and findings suggesting ischemia who presented for clinically indicated coronary angiography to further evaluate suspected myocardial ischemia, as described in detail previously (12). An IVUS sub-study was conducted in a subgroup of 100 women without significant luminal narrowing $(>50 \%)$ and defined as having non-obstructive CAD (13). For the purpose of the current analysis, 87 of these women had IVUS images satisfactory of detailed analysis of remodelling indices and matchable by age and ethnicity to women with obstructive CAD on angiography selected from a clinical trials database had employed serial evaluations of plaque progression using IVUS (15-18). In addition to age and ethnicity match, the latter women were required to be symptomatic and have at least one obstructive stenosis on an angiogram. All participants provided written informed consent prior to enrollment.

\section{Acquisition and analysis of IVUS images}

IVUS images were acquired and analyzed as described in detail previously $(13,15-18)$. In brief, a catheter containing a high frequency (30-40 MHz) ultrasound transducer on the tip was placed as distally as possible within the coronary artery selected for evaluation following anticoagulation and administration of intracoronary nitroglycerin. For patients with non-obstructive CAD the longest and least angulated vessel was selected for analysis. For patients with obstructive $\mathrm{CAD}$ it was required that the target vessel contained a segment of at least $30 \mathrm{~mm}$ in length with no stenosis greater than $50 \%$. Continuous ultrasonic imaging was performed during withdrawal of the catheter by an automatic pullback device at a rate of $0.5 \mathrm{~mm} / \mathrm{s}$ and recorded on videotape at 30 frames/s. All images were analyzed using the same protocol by investigators in the Intravascular Ultrasound Core Laboratory at the Cleveland Clinic. The longest arterial segment, whose limits were defined by the presence of a proximal and distal side branch, were chosen for analysis. Cross-sectional images, spaced $1 \mathrm{~mm}$ apart, were selected for analysis. The leading edges of the external elastic membrane (EEM) and lumen were traced by manual 
planimetry using the National Institutes of Health Image J (version 1.62, National Institutes of Health public domain software, Bethesda, MD).

Calculation of the total atheroma volume (TAV) was performed by summation of plaque areas, defined as the difference between EEM and lumen area, of all evaluable images (19).

Normalized TAV $=\frac{\sum\left(\mathrm{EEM}_{\text {area }}-\text { Lumen }_{\text {area }}\right)}{\text { Number of images in pullback }} \times$ Median number of imagesin cohort

To account for variability in the length of segment evaluated in different subjects TAV, total lumen volume and total EEM were normalized by adjusting for the median number of images in the entire study population. Normalized volumes occupied by the EEM and lumen were determined in a similar fashion. Percent atheroma volume $(\mathrm{PAV})$ was calculated as the percentage of the sum of EEM areas occupied by TAV (19).

$$
\mathrm{PAV}=\frac{\sum\left(\mathrm{EEM}_{\text {area }}-\text { Lumen }_{\text {area }}\right)}{\sum \mathrm{EEM}_{\text {acea }}} \times 100
$$

Atheroma burden was also expressed as the percentage of images containing plaque, defined as a maximal plaque thickness greater than $0.5 \mathrm{~mm}$.

The arterial remodelling index at the site containing the greatest plaque area in each pullback was also determined as the ratio of the EEM area at that site compared with the EEM area at a reference point, defined as the slice within the proximal $10 \mathrm{~mm}$ that contained the least amount of plaque. In accordance with consensus guidelines, remodelling at the most diseased site was defined as constrictive (index $<0.95$ ) or expansive (index $>1.05$ ) according to the remodelling index (20).

\section{Statistical analysis}

All statistical analyses were performed using SAS software version 8.2 (SAS Inc., Cary NC). Continuous variables are expressed as mean \pm standard deviation (SD) and categorical variables as percentage. Comparisons between matched groups were performed using chi-square tests for categorical variables and $t$-tests for continuous variables. An analysis of covariance (ANCOVA) model was used to compare IVUS measures between groups adjusting for differences in clinical characteristics (congestive heart failure, hypertension, metabolic syndrome, previous myocardial infarction, lowdensity lipoprotein (LDL) cholesterol, triglyceride and diastolic blood pressure). Adjusted measures are expressed as least square mean \pm standard error of the mean (SEM). A $P$ value $<0.05$ was considered significant.

\section{Results}

\section{Clinical characteristics}

Pertinent clinical characteristics of the women undergoing coronary angiography for suspected ischemia with and without obstructive CAD on coronary angiography are summarized in Table 1. For reference purposes, the women with non-obstructive CAD had an angiographic CAD severity index $7.0 \pm 3.8$, calculated based on the stenosis severity weighted by proximal lesion as previously published (21). Women with obstructive $\mathrm{CAD}$ had a more atherogenic risk factor profile with greater rates of hypertension $(86.2 \% \mathrm{vs}$. $36.0 \%, \mathrm{P}<0.001$ ), smoking (38.8\% vs. $13.8 \%, \mathrm{P}<0.001$ ), hyperlipidemia $(77.0 \%$ vs. $31.0 \%, \mathrm{P}<0.001)$, metabolic syndrome $(62.1 \%$ vs. $34.6 \%, \mathrm{P}<0.001)$ and previous myocardial infarction $(32.2 \%$ vs. $7.1 \%, \mathrm{P}<0.001)$. Women with obstructive CAD also had higher levels of total cholesterol $(212.7 \pm 46.1$ vs. $182.5 \pm 33.0 \mathrm{mg} / \mathrm{dL}, \mathrm{P}<0.001)$, LDL cholesterol $(126.4 \pm 39.4$ vs. $102.3 \pm 37.3 \mathrm{mg} / \mathrm{dL}$, $\mathrm{P}<0.001)$, triglyceride $(182.9 \pm 87.4$ vs. $99.6 \pm 79.9 \mathrm{mg} / \mathrm{dL}$, $\mathrm{P}<0.001)$ and diastolic blood pressure $(77.5 \pm 10.9$ vs. $74.5 \pm 10.7 \mathrm{mmHg}, \mathrm{P}=0.080$ ), although the later failed to meet statistical significance.

\section{Atheroma burden}

Measures of the extent of coronary atherosclerosis in women with and without obstructive CAD are summarized in Table 2. All indices of atheroma burden were greater in women with obstructive $\mathrm{CAD}$ on angiography including PAV $(36.1 \% \pm 9.8 \%$ vs. $25.4 \% \pm 9.1 \%, \mathrm{P}<0.0001)$, TAV $\left(140.8 \pm 58.7\right.$ vs. $\left.98.8 \pm 46.9 \mathrm{~mm}^{3}, \mathrm{P}<0.0001\right)$ and the percentage of images containing plaque $(70.0 \% \pm 30.5 \%$ vs. $35.7 \% \pm 32.6 \%, \mathrm{P}<0.0001)$. This distinction remained significant following adjustment for differences between the groups with regard to clinical characteristics.

\section{Epicardial coronary arterial wall remodelling}

Indices of coronary arterial remodelling, throughout the entire segment and at the site containing the greatest amount of plaque in women with and without obstructive CAD on angiography, are summarized in Table 2. Women 
Table 1 Clinical characteristics by absence and presence of obstructive CAD

\begin{tabular}{|c|c|c|c|}
\hline Characteristic & Non-obstructive CAD $(n=87)$ & Obstructive CAD ( $\mathrm{n}=87)$ & $P$ value \\
\hline Caucasian (\%) & 87.4 & 87.4 & N/A \\
\hline Body mass index $\left(\mathrm{kg} / \mathrm{m}^{2}\right)$ & $31.9 \pm 8.8$ & $32.2 \pm 7.7$ & 0.780 \\
\hline Diabetes (\%) & 17.2 & 20.7 & 0.560 \\
\hline Current smoker (\%) & 13.8 & 38.8 & $<0.001$ \\
\hline Hyperlipidemia (\%) & 31.0 & 77.0 & $<0.001$ \\
\hline Metabolic syndrome (\%) & 34.6 & 62.1 & $<0.001$ \\
\hline Previous myocardial Infarction (\%) & 7.1 & 32.2 & $<0.001$ \\
\hline Total cholesterol (mg/dL) & $182.5 \pm 33.0$ & $212.7 \pm 46.1$ & $<0.001$ \\
\hline LDL cholesterol (mg/dL) & $102.3 \pm 37.3$ & $126.4 \pm 39.4$ & $<0.001$ \\
\hline HDL cholesterol (mg/dL) & $50.5 \pm 13.0$ & $49.8 \pm 11.3$ & 0.920 \\
\hline Triglyceride (mg/dL) & $99.6 \pm 79.9$ & $182.9 \pm 87.4$ & $<0.001$ \\
\hline
\end{tabular}

Continuous variables expressed as mean $\pm \mathrm{SD}$ and categorical variables expressed as percentage. CAD, coronary artery disease; LDL, low-density lipoprotein; HDL, high-density lipoprotein; SD, standard deviation; N/A, not available.

Table 2 Atheroma burden and remodelling parameters by absence and presence of obstructive CAD

\begin{tabular}{|c|c|c|c|c|}
\hline Characteristic & Non-obstructive CAD $(n=87)$ & Obstructive CAD ( $\mathrm{n}=87)$ & $P$ value & Adjusted $\mathrm{P}$ value* \\
\hline Total atheroma volume $\left(\mathrm{mm}^{3}\right)$ & $98.8 \pm 46.9$ & $140.8 \pm 58.7$ & $<0.0001$ & 0.110 \\
\hline Percent images containing plaque (\%) & $35.7 \pm 32.6$ & $70.0 \pm 30.5$ & $<0.0001$ & 0.0008 \\
\hline EEM volume $\left(\mathrm{mm}^{3}\right)$ & $388.6 \pm 113.7$ & $392.7 \pm 128.1$ & 0.910 & 0.400 \\
\hline Remodelling index & $0.96 \pm 0.25$ & $0.96 \pm 0.20$ & 0.89 & - \\
\hline Constrictive remodelling (\%) & 40.2 & 46.4 & 0.41 & - \\
\hline Expansive remodelling (\%) & 25.3 & 25.0 & 0.97 & - \\
\hline
\end{tabular}

Measures of arterial wall remodelling throughout the segment of vessel evaluated and at the site containing the greatest plaque area in age and ethnicity matched females without and with obstructive CAD. Univariate results expressed as mean \pm SD. Multivariate results expressed as least square mean \pm SEM. *, adjusted for differences in clinical characteristics (congestive heart failure, hypertension, metabolic syndrome, previous myocardial infarction, LDL cholesterol, triglyceride and diastolic blood pressure). CAD, coronary artery disease; LDL, low-density lipoprotein; EEM, external elastic membrane; SEM, standard error of the mean; SD, standard deviation.

with obstructive CAD had smaller lumen volumes

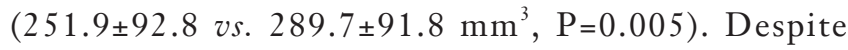
the presence of more extensive atherosclerosis, women with obstructive CAD did not have a greater volume occupied by the EEM $\left(392.7 \pm 128.1\right.$ vs. $388.6 \pm 113.7 \mathrm{~mm}^{3}$, $\mathrm{P}=0.910$ ). The difference in lumen, but not EEM, volume remained following adjustment for differences in clinical characteristics between the groups. In contrast, no 
Table 3 Atheroma burden and arterial remodelling in subjects matched according to total atheroma volume by absence and presence of obstructive CAD

\begin{tabular}{lcc}
\hline Characteristic & Non-obstructive CAD & Obstructive CAD \\
\hline Total atheroma volume $\left(\mathrm{mm}^{3}\right)$ & $107.0 \pm 41.1$ & $107.7 \pm 40.8$ \\
Percent atheroma volume (\%) & $27.5 \pm 7.9$ & $32.0 \pm 10.0$ \\
Percent images containing plaque (\%) & $39.9 \pm 31.2$ & $53.7 \pm 31.8$ \\
EEM volume $\left(\mathrm{mm}^{3}\right)$ & $391.3 \pm 105.6$ & $343.3 \pm 92.9$ \\
Lumen volume $\left(\mathrm{mm}^{3}\right)$ & $284.3 \pm 85.9$ & $235.7 \pm 79.8$ \\
Remodelling index & $0.99 \pm 0.26$ & $0.97 \pm 0.26$ \\
Constrictive remodelling (\%) & 34.4 & 45.0 \\
Expansive remodelling (\%) & 26.2 & 31.7 \\
\hline
\end{tabular}

Continuous variables expressed as mean $\pm \mathrm{SD}$ and categorical variables expressed as percentage. CAD, coronary artery disease; EEM, external elastic membrane; SD, standard deviation.

difference was observed between the groups with regard to the remodelling index or percentage of subjects undergoing constrictive or expansive remodelling at the site containing the greatest amount of plaque.

To further explore this difference in remodelling pattern throughout the evaluated arterial segment, groups were also compared following additional matching for TAV (Table 3). Women with obstructive CAD ( $\mathrm{n}=61)$ continued to demonstrate greater levels of PAV $(32.0 \% \pm 10.0 \%$ vs. $27.5 \% \pm 7.9 \%, \mathrm{P}=0.007)$ and percentage of images containing plaque $(53.7 \% \pm 31.8 \%$ vs. $39.9 \% \pm 31.2 \%, \mathrm{P}=0.020)$. Women with obstructive $\mathrm{CAD}$ had smaller volumes occupied not only by the lumen $\left(235.7 \pm 79.8 v\right.$ s. $\left.284.3 \pm 85.9 \mathrm{~mm}^{3}, \mathrm{P}=0.002\right)$ but also the EEM $\left(343.3 \pm 92.9\right.$ vs. $391.3 \pm 105.6 \mathrm{~mm}^{3}$, $\mathrm{P}=0.010)$. Among the women without obstructive $\mathrm{CAD}$, the correlation between $\mathrm{EEM}$ volume and $\mathrm{PAV}$ was $\mathrm{r}=0.82(\mathrm{P}<0.05)$, supportive of a continuous process of EEM expansion in the lesions with larger PAV in non-obstructive CAD.

\section{Discussion}

Ultrasonic coronary artery imaging provides a unique opportunity to study the natural history of atherosclerosis in vivo using a quantitative analytical approach that can be applied to elucidate factors influencing the pathogenesis of IHD. We have previously shown, in a sample of women from the WISE without obstructive CAD on angiography, that most $(\sim 80 \%)$ had evidence of coronary artery atherosclerosis by IVUS and that plaque was concealed on angiography by negative remodelling in a subgroup (13). However, those findings were difficult to interpret in the absence of a comparison group of women. In the current study, IVUS data from the WISE sample of women with symptoms and signs of ischemia without obstructive CAD was compared with a group of women with symptoms and signs of ischemia with obstructive CAD on angiography matched by age and ethnicity. Both groups were analysed by the same IVUS core lab masked to clinical and angiographic findings. While no direct implication can be made with regard to chronicity of the disease process in these women studied cross-sectionally, our finding that women with obstructive CAD harboured considerably more, as well as, more extensive coronary atheroma is consistent with an evolving disease process which results in the appearance mild atheroma without luminal narrowing and then luminal narrowings associated with increasing accumulation of plaque. The current findings provide important insight into the relationship between plaque deposition and the coronary arterial remodelling response, as well as the appearance on angiography.

The arterial wall is not a passive participant in the development and accumulation of atherosclerotic plaque. Rather, considerable evidence suggests that the coronary artery wall undergoes changes in size and shape, termed remodelling, in response to both progression and regression of atherosclerosis. The initial report of remodelling, on the basis of necropsy specimens, to early atheroma accumulation is of expansion of the EEM (22). This is associated with a relative preservation of lumen dimension and provides a rationale for accumulation of substantial atheroma, despite the presence of a normal angiogram, in many patients. The findings of the current analysis may 

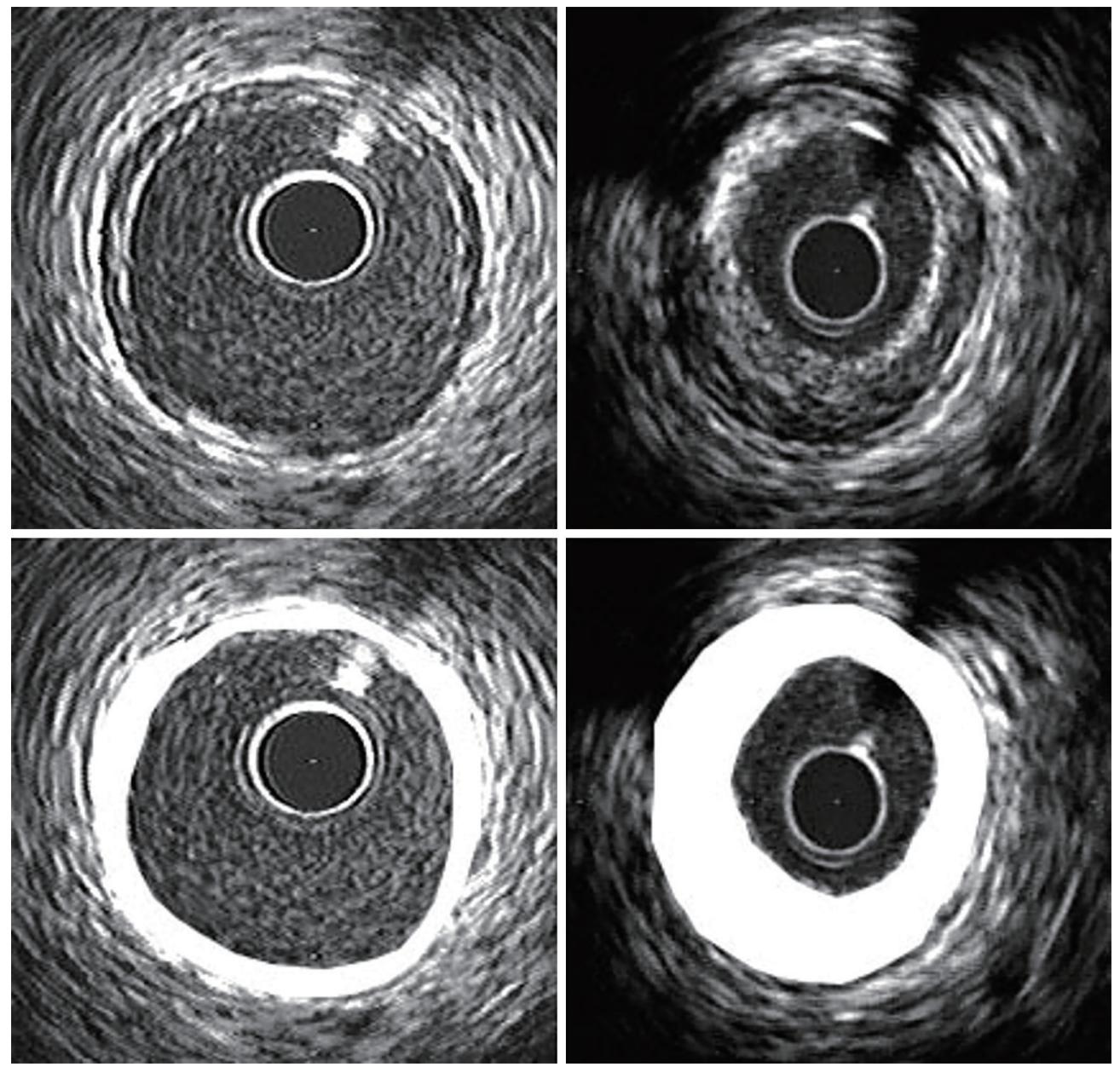

Figure 1 Illustrative example of differences in remodelling between a female subject with symptomatic ischemia in the absence (left panels) or presence (right panels) of obstructive disease on coronary angiography. Location of atherosclerotic plaque in cross-sectional images is depicted by shading in the lower panels. A lack of compensatory expansion of the outer vessel border in the presence of a greater plaque burden results in a smaller lumen, which would be detected as an abnormality on angiography.

provide further insight into the role of coronary arterial remodelling in the pathogenesis of atherosclerotic CAD in women. It is of particular interest to note that there was no difference between the women with and without obstructive CAD with regard to the volume occupied by the EEM despite a significant difference in atheroma volume (Figure 1). The consequence of this inadequate compensatory remodelling of the coronary artery in the presence of more extensive atherosclerosis is the contraction of lumen dimensions, which appears as obstructive CAD on angiography. Potential differences between the groups of women are further emphasized by the finding that when subjects were matched on the basis of atheroma volume, the absence of obstructive CAD was accompanied by larger
EEM and lumen volumes.

A large number of studies have employed IVUS to characterize patterns of coronary arterial remodelling (14). These investigations have confirmed the typical pattern of expansive remodelling of the EEM in the early stages of plaque accumulation. The relationship between expansive remodelling of culprit lesions, elevated systemic metalloproteinases and acute ischemic syndromes highlights further potential mechanistic links between remodelling and the clinical expression of disease $(23,24)$. The current results expand these observations to include study of women with suspected ischemia but non-obstructive $\mathrm{CAD}$ and support the hypothesis that compensatory arterial remodelling may be prevalent in women. Further prospective studies, as well as sex-specific 
comparative studies, are clearly needed in this area.

More recent studies, mostly among men, have utilized serial evaluations of coronary arteries by IVUS to evaluate the impact of medical therapies on atheroma progression rates (15-18,25-29). Of interest, regression of atherosclerotic plaque is associated with a pattern of reverse remodelling, characterized by contraction of the EEM and relative preservation of lumen dimensions (30). In a pooled analysis of these studies, it was suggested that women harbored less atherosclerotic plaque, despite the presence of a more atherogenic risk factor profile than observed in men from these studies (31). The finding that women had smaller vessel volumes following correction for body size was consistent with different patterns of remodelling between women and men at baseline (31). More recently, it has been reported that there are no differences between genders with regard to the pattern of reverse remodelling that accompanies plaque regression (32).

A number of caveats should be noted with regard to our current analysis. Given the requirement for invasive catheterisation of the coronary arteries, all of the women had presented for a clinically indicated coronary angiogram for suspected myocardial ischemia that creates potential for an indication bias. It is uncertain whether current findings can be extrapolated to asymptomatic women. A treatment bias may have influenced risk factor status, particularly among women with an established diagnosis of obstructive CAD. Specifically, women with obstructive CAD were more likely to receive statin therapy and this may have also had a direct impact on vessel wall remodelling. A segment of only one coronary artery was evaluated in each woman: it is unknown whether the data confidently reflects findings throughout the entire coronary arterial tree. Also, of note, some segments of the women with obstructive CAD contained no stenosis $>50 \%$, and therefore uncertain whether the current distinction applies to women with more severe forms of obstructive CAD. IVUS provides a limited characterization of plaque components and therefore it is not possible to accurately investigate relationships between plaque composition and arterial remodelling in these two groups of women.

In summary, the current findings suggest that among women referred for suspected ischemia, there appear to be differing patterns of coronary arterial response to accumulation of atherosclerosis, and compensatory remodelling related to the presence and absence of obstructive CAD. The presence of obstructive CAD on angiography is associated with more extensive atherosclerosis and an inadequate compensatory arterial remodelling response of the arterial wall. The inability of the outer vessel wall to expand likely results in relative lumen contraction and the appearance of obstructive CAD. Combined with the relatively high rates of nonobstructive CAD in the setting of ischemia among women undergoing coronary angiography, these findings suggest that compensatory coronary arterial remodelling may be prevalent in women. Preservation and cultivation of compensatory arterial remodelling may be a novel CAD therapeutic target.

\section{Acknowledgements}

The technical expertise of the Intravascular Ultrasound Core Laboratory at the Cleveland Clinic is appreciated.

The WISE study was sponsored by the National Heart, Lung, and Blood Institute of the National Institute of Health. This work was supported by contracts from the National Heart, Lung and Blood Institutes, nos. N01HV-68161, N01-HV-68162, N01-HV-68163, N01HV-68164, a GCRC grant MO1-RR00425 from the National Center for Research Resources, and grants from the Gustavus and Louis Pfeiffer Research Foundation, Denville, New Jersey, The Women's Guild of CedarsSinai Medical Center, Los Angeles, California, The Ladies Hospital Aid Society of Western Pennsylvania, Pittsburgh, Pennsylvania, the Edythe Broad Endowment for Women's Heart Disease Research, Los Angeles, California, and the Barbra Streisand Endowment for Women's Heart Disease Research and Education Program, Los Angeles, California. The ACTIVATE study was sponsored by Sankyo Pharmaceuticals. The ASTEROID study was sponsored by AstraZeneca Pharmaceuticals. The CAMELOT and REVERSAL studies were sponsored by Pfizer Pharmaceuticals.

\section{Footnote}

Conflicts of Interest: Dr. CN Bairey Merz reports personal honoraria and consulting from ACRWH (NIH Advisory Council), NIH-CASE (grant review study section), Springer International (book honorarium), Decision Support in Medicine LLC (book honorarium), and NHLBI Research Triangle Institute (RTI) International, as well as personal research grants for WISE HFpEF, RWISE, FAMRI, DoD, 
and California Institute for Precision Medicine. The other authors have no conflicts of interest to declare.

Ethical Statement: The study was approved by institutional/ regional/national ethics/committee/ethics board of the University of Florida (No. 201602567) and informed consent was taken from all the patients.

\section{References}

1. Association AH. Heart disease and stroke statistics - 2003 update. Dallas, Tex: AHA, 2002.

2. Benjamin EJ, Smith SC Jr, Cooper RS, et al. Task force \#1--magnitude of the prevention problem: opportunities and challenges. 33rd Bethesda Conference. J Am Coll Cardiol 2002;40:588-603.

3. Mosca L, Grundy SM, Judelson D, et al. Guide to Preventive Cardiology for Women.AHA/ACC Scientific Statement Consensus panel statement. Circulation 1999;99:2480-4.

4. Association AH. Heart disease and stroke statistics - 2004 update. Dallas, Tex: AHA, 2003.

5. Bowling A, Bond $\mathrm{M}, \mathrm{McKee} \mathrm{D}$, et al. Equity in access to exercise tolerance testing, coronary angiography, and coronary artery bypass grafting by age, sex and clinical indications. Heart 2001;85:680-6.

6. Epstein AM, Weissman JS, Schneider EC, et al. Race and gender disparities in rates of cardiac revascularization: do they reflect appropriate use of procedures or problems in quality of care? Med Care 2003;41:1240-55.

7. Mosca L, Appel LJ, Benjamin EJ, et al. Evidence-based guidelines for cardiovascular disease prevention in women. Circulation 2004;109:672-93.

8. Rathore SS, Wang Y, Radford MJ, et al. Sex differences in cardiac catheterization after acute myocardial infarction: the role of procedure appropriateness. Ann Intern Med 2002;137:487-93.

9. Pepine CJ, Kerensky RA, Lambert CR, et al. Some thoughts on the vasculopathy of women with ischemic heart disease. J Am Coll Cardiol 2006;47:S30-5.

10. Bairey Merz CN, Shaw LJ, Reis SE, et al. Insights from the NHLBI-Sponsored Women's Ischemia Syndrome Evaluation (WISE) Study: Part II: gender differences in presentation, diagnosis, and outcome with regard to gender-based pathophysiology of atherosclerosis and macrovascular and microvascular coronary disease. J Am Coll Cardiol 2006;47:S21-9.

11. Jespersen L, Hvelplund A, Abildstrøm SZ, et al. Stable angina pectoris with no obstructive coronary artery disease is associated with increased risks of major adverse cardiovascular events. Eur Heart J 2012;33:734-44.

12. Merz CN, Kelsey SF, Pepine CJ, et al. The Women's Ischemia Syndrome Evaluation (WISE) study: protocol design, methodology and feasibility report. J Am Coll Cardiol 1999;33:1453-61.

13. Khuddus MA, Pepine CJ, Handberg EM. An intravascular ultrasound analysis in women experiencing chest pain in the absence of obstructive coronary artery disease: a substudy from the National Heart, Lung and Blood Institute-Sponsored Women's Ischemia Syndrome Evaluation (WISE). J Interv Cardiol 2010;23:511-9.

14. Schoenhagen P, Ziada KM, Vince DG, et al. Arterial remodeling and coronary artery disease: the concept of "dilated" versus "obstructive" coronary atherosclerosis. J Am Coll Cardiol 2001;38:297-306.

15. Nissen SE, Tuzcu EM, Brewer HB, et al. Effect of ACAT inhibition on the progression of coronary atherosclerosis. N Engl J Med 2006;354:1253-63.

16. Nissen SE, Nicholls SJ, Sipahi I, et al. Effect of very high-intensity statin therapy on regression of coronary atherosclerosis: the ASTEROID trial. JAMA 2006;295:1556-65.

17. Nissen SE, Tuzcu EM, Libby P, et al. Effect of antihypertensive agents on cardiovascular events in patients with coronary disease and normal blood pressure: the CAMELOT study: a randomized controlled trial. JAMA 2004;292:2217-25.

18. Nissen SE, Tuzcu EM, Schoenhagen P, et al. Effect of intensive compared with moderate lipid-lowering therapy on progression of coronary atherosclerosis: a randomized controlled trial. JAMA 2004;291:1071-80.

19. Nicholls SJ, Sipahi I, Schoenhagen P, et al. Intravascular ultrasound assessment of novel antiatherosclerotic therapies: rationale and design of the Acyl-CoA:Cholesterol Acyltransferase Intravascular Atherosclerosis Treatment Evaluation (ACTIVATE) Study. Am Heart J 2006;152:67-74.

20. Mintz GS, Nissen SE, Anderson WD, et al. American College of Cardiology Clinical Expert Consensus Document on Standards for Acquisition, Measurement and Reporting of Intravascular Ultrasound Studies (IVUS). A report of the American College of Cardiology Task Force on Clinical Expert Consensus Documents. J Am Coll Cardiol 2001;37:1478-92.

21. Sharaf BL, Pepine CJ, Kerensky RA, et al. Detailed angiographic analysis of women with suspected ischemic 
chest pain (pilot phase data from the NHLBI-sponsored Women's Ischemia Syndrome Evaluation [WISE] Study Angiographic Core Laboratory). Am J Cardiol 2001;87:937-41; A3.

22. Glagov S, Weisenberg E, Zarins CK, et al. Compensatory enlargement of human atherosclerotic coronary arteries. N Engl J Med 1987;316:1371-5.

23. Schoenhagen P, Vince DG, Ziada KM, et al. Relation of matrix-metalloproteinase 3 found in coronary lesion samples retrieved by directional coronary atherectomy to intravascular ultrasound observations on coronary remodeling. Am J Cardiol 2002;89:1354-9.

24. Schoenhagen P, Vince DG, Ziada KM, et al. Association of arterial expansion (expansive remodeling) of bifurcation lesions determined by intravascular ultrasonography with unstable clinical presentation. Am J Cardiol 2001;88:785-7.

25. Nissen SE, Nicholls SJ, Wolski K, et al. Comparison of pioglitazone vs glimepiride on progression of coronary atherosclerosis in patients with type 2 diabetes: the PERISCOPE randomized controlled trial. JAMA 2008;299:1561-73.

26. Nissen SE, Tardif JC, Nicholls SJ, et al. Effect of torcetrapib on the progression of coronary atherosclerosis. N Engl J Med 2007;356:1304-16.

Cite this article as: Nicholls SJ, Tuzcu EM, Wolski K, Johnson BD, Sopko G, Sharaf BL, Pepine CJ, Nissen SE, Bairey Merz $\mathrm{CN}$. Extent of coronary atherosclerosis and arterial remodelling in women: the NHLBI-sponsored Women's Ischemia Syndrome Evaluation. Cardiovasc Diagn Ther 2018;8(4):405413. doi: 10.21037/cdt.2018.04.03
27. Nissen SE, Nicholls SJ, Wolski K, et al. Effect of rimonabant on progression of atherosclerosis in patients with abdominal obesity and coronary artery disease: the STRADIVARIUS randomized controlled trial. JAMA 2008;299:1547-60.

28. Tardif JC, Gregoire J, L'Allier PL, et al. Effects of the acyl coenzyme A:cholesterol acyltransferase inhibitor avasimibe on human atherosclerotic lesions. Circulation 2004;110:3372-7

29. Tardif JC, Gregoire J, L'Allier PL, et al. Effects of reconstituted high-density lipoprotein infusions on coronary atherosclerosis: a randomized controlled trial. JAMA 2007;297:1675-82.

30. Nicholls SJ, Tuzcu EM, Sipahi I, et al. Relationship between atheroma regression and change in lumen size after infusion of apolipoprotein A-I Milano. J Am Coll Cardiol 2006;47:992-7.

31. Nicholls SJ, Wolski K, Sipahi I, et al. Rate of progression of coronary atherosclerotic plaque in women. J Am Coll Cardiol 2007;49:1546-51.

32. Kalidindi SR, Hsu A, Moon KW, et al. Impact of gender on serial atherosclerotic changes in response to established medical therapies. Circulation 2007;116:II_235. 